\title{
An efficient methodology for the experimental characterization of mode II delamination growth under fatigue loading
}

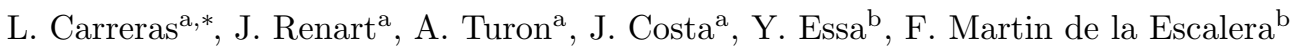 \\ ${ }^{a}$ AMADE, Polytechnic School, University of Girona, Campus Montilivi s/n, E-17003 Girona, Spain \\ ${ }^{b}$ AERNNOVA Engineering Division SAU, Parque Tecnológico de Álava, Leonardo Da Vinci 13, E-01510 Álava, Spain
}

\begin{abstract}
Crack growth rate curves provide information about the delamination resistance of composite materials under cyclic loading. The existing methodologies for mode II fatigue testing using three-point bending end-notched flexure (3-ENF) under constant cyclic displacement conditions yield discontinuous delamination growth rate curves, therefore requiring a batch of several specimens to be tested under different severity conditions in order to fully characterize the crack growth. This work describes a variable cyclic displacement test procedure that, in combination with the real time monitoring of the specimen's compliance, allows the crack growth rate to be measured for the desired range of severities with a single specimen, thus avoiding any human intervention during the test.
\end{abstract}

Keywords:

Mode II, Delamination, Testing method, Fatigue, 3-ENF, Composites

1 1. Introduction

${ }^{*}$ Corresponding author. Tel.: +34972 418817

Email addresses: laura.carreras@udg.edu (L. Carreras), jordi.renart@udg.edu (J. Renart), albert.turon@udg.edu (A. Turon), josep.costa@udg.edu (J. Costa), yasser.essa@aernnova.com (Y. Essa), federico.martindelaescalera@aernnova.com (F. Martin de la Escalera) 
will ever occur over the lifetime of the component. This approach relies on fatigue onset tests [2] which, for a given load intensity, determine the number of cycles required to make a crack grow perceptibly. Conversely, the damage tolerance approach is based on a structure's remaining capacity to safely sustain in-service loads, even with the presence of sub-critical sized delaminations. That is, crack growth is allowed provided that it does not reach an unsafe size during service. To this end, crack propagation tests evaluate the crack growth rate $(\mathrm{d} a / \mathrm{d} N$ ) as a function of the severity of load. The severity of load is usually defined as the ratio of the maximum energy release rate of the cycle $\left(\mathcal{G}_{m a x}\right)$ to the quasi-static fracture toughness $\mathcal{G}_{c}[3-7]$, although other expressions also exist in the literature.

The onset and propagation of interlaminar cracks are experimentally characterized for the different loading modes of propagation (I, II or III). Delamination growth under mode I loading is usually assessed with double cantilever beam (DCB) specimens, while for mode II the End Notched Flexure (3-ENF) test performed under displacement control with sinusoidal shaped loading cycles of constant displacement is the most widely employed (see figure 1a) [3, 4, 8-11]. Other mode II test set-ups include the calibrated end-loaded split (C-ELS) [12] and the four-point bending end-notched flexure (4-ENF). Either the C-ELS [10] and the 4-ENF [13] have been used for delamination resistance testing under fatigue. However, while the 4-ENF test is not preferable because of friction effects [14], only further research will show whether 3-ENF or C-ELS is better suited for cyclic mode II fatigue delamination characterization [10].

a)

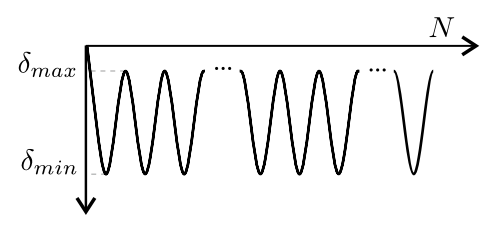

b)

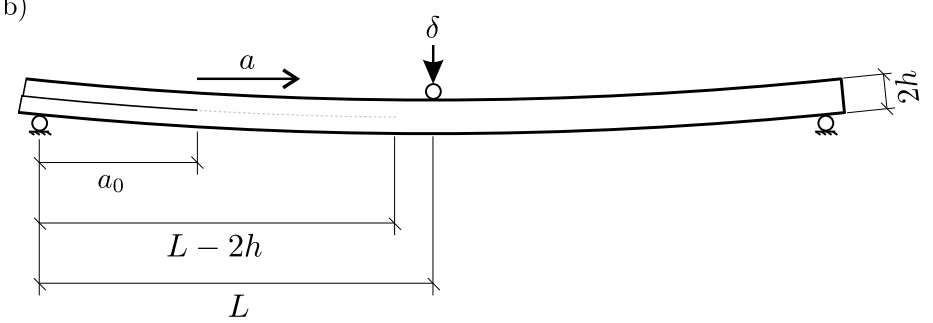

Figure 1: a) Sinusoidal shaped loading cycles with constant displacement. The sign convention used in this work is negative for displacements which result in compressive reaction forces. b) 3-ENF test configuration, where $L$ is the mid-span length, $a$ is the crack length, $a_{0}$ is the initial crack length and $2 h$ is the specimen's total thickness of the specimen. 
The selection of the testing parameters for mode I fatigue experiments is not critical. For a test conducted under displacement control and constant displacement amplitude, the energy release rate (the severity of the load) decreases as the crack grows. That is, the crack growth rate vs. load severity curve sweeps from left to right until the crack growth rate becomes unnoticeable (the threshold, the severity for which the crack growth rate tends to zero). Nevertheless, even in mode I, the determination of the threshold value remains elusive due to the need of high sensitivity measurement devices to capture the actual growth rates [15].

For mode II 3-ENF experiments the contrary applies as the range of the crack growth rate curve swept in a single test under constant cyclic displacement is very narrow. This results from the dependence of the energy release rate on the geometry of the specimen and the configuration of the test. Indeed, in 3-ENF tests the region available for crack propagation spans between the support and the vicinity of the loading roller, where the through-thickness compression arrests crack propagation. Previous studies [16] estimate that when the crack tip approaches the loading point by $2 h$ the experiment is no longer valid; being $2 h$ the laminate thickness (see figure $1 \mathrm{~b}$ ). The energy release rate does not evolve monotonically with the crack extension but, as the cracks extends, it increases and then decreases, as shown in figure 2a. Thus, only a small segment of the crack growth curve is covered by a single test. In fact, the same segment of the curve is tracked twice: first upward and then downward (figure $2 \mathrm{~b}$ ). In addition, detecting the threshold becomes practically unfeasible. Hence, to construct the entire crack growth curve requires various constant cyclic displacement tests at different load intensities $[3,8-10]$. The alternative to performing multiple tests is to implement a test with a proper variation of the displacement, which is what this manuscript focuses on.

Preceding the methodology presented in this work, only Tanaka and Tanaka [17], Matsubara et al. [18] and Hojo et al. [19, 20] carried out 3-ENF fatigue tests with fiber reinforced polymer specimens by decreasing the applied peak load as the crack propagates. In [17] the authors conducted fatigue tests under either a constant or decreasing stress intensity range, $\Delta K$, to graphite/epoxy composite specimens. The same data on crack growth rate, $\mathrm{d} a / \mathrm{d} N$, with crack extension was obtained in the $\Delta K$ - 
a)

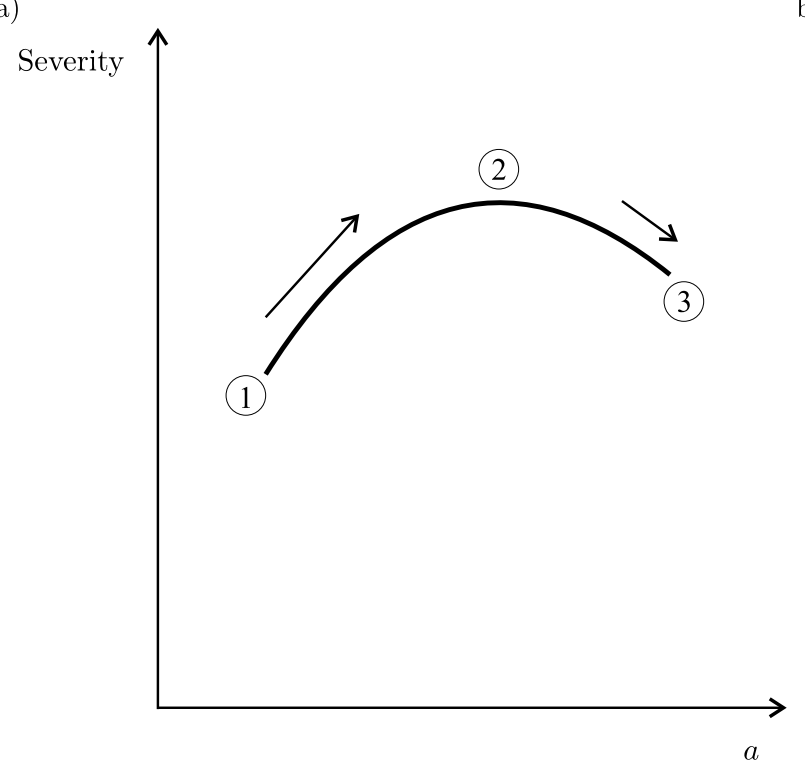

b)

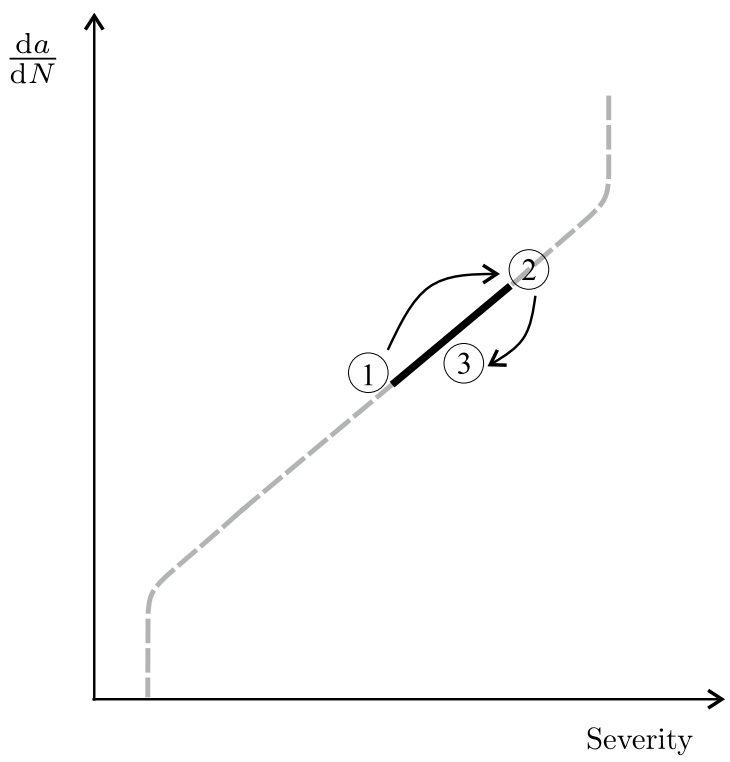

Figure 2: a) Maximum energy release rate applied to the 3-ENF test as a function of the crack length. b) Crack growth rate curve segment analyzed in a single test with constant cyclic displacement.

constant tests. Tanaka and Tanaka determined that fiber bridging had no influence on Mode II crack propagation and concluded that crack growth is independent of crack extension history. Matsubara et al. [18] used a decreasing-load test procedure based on the ASTM standard for metals [21]. This methodology enables the crack growth rate for a broader range of the load intensity factor to be determined and also to approach the low-rate region, near the threshold, by decreasing the applied load. The load shedding can be done manually at selected crack size intervals or, alternatively, by continuously reducing the force to adjust the normalized $K$-gradient, $(1 / K) \mathrm{d} K / \mathrm{d} a$, to a fixed value. They conducted constant- and decreasing- load tests with glass fiber reinforced polymer specimens and obtained identical results, confirming that crack growth rate is independent of crack extension history. Similarly, Hojo et al. [19, 20] carried out fatigue tests under constant normalized gradient of energy release rate, $(1 / \mathcal{G}) \mathrm{d} \mathcal{G} / \mathrm{d} a$, by measuring the specimen's compliance and decreasing the peak load accordingly.

In practice, incrementally shedding the force with increasing crack size requires the continuous intervention of a technician. On the other hand, computer-controlled stress intensity or, equivalently, energy release rate gradient techniques $[22,23]$, require the crack length to be monitored in real-time, 
usually by means of the specimen's compliance. Indeed, the use of the compliance to control the machine, or any other behavioral-based control technology, can lead to unexpected load setpoints.

This work presents a methodology to measure, in a single test, a larger region of the crack growth rate curve than that achieved in a constant cyclic displacement test. The procedure consists of varying the cyclic applied displacement, $\delta_{\min }$ and $\delta_{\max }$, while keeping the displacement ratio, $R$, constant. Moreover, the displacement variation is calculated a priori, so that it can be implemented in the control software of the testing machine. Thus, the control loop does not make use of parameters related to the behavior of the specimen, which could lead to unpredictable responses from the test machine. This method requires neither human intervention during the test nor processing the data in real time. The manuscript includes a test campaign carried out on carbon fiber reinforced composites which exemplifies the advantages of the proposed procedure: the crack growth rate curve can be characterized in, at most, 1/80 of the time required for a constant cyclic displacement test.

\section{Methodology}

Due to the geometry of the test, the maximum energy release rate during one load cycle, $\mathcal{G}_{\max }$ corresponds to the minimum displacement, $\delta_{m i n}$. The aim of the proposed methodology is to define the evolution of $\delta_{\min }$ with the number of cycles, $N$, so that the severity of the load sweeps a predefined range, from $\mathcal{G}_{\max , 0} / \mathcal{G}_{c}$ to $\mathcal{G}_{\max , f} / \mathcal{G}_{c}$, while the crack grows from the initial crack, $a_{0}$, to the maximum allowed crack length, $a_{f}$, (when the crack tip approaches a distance $2 h$ from the load introduction point). $\delta_{\max }$ is established so that the $R$-ratio is constant throughout the fatigue test $\left(R=\delta_{\min } / \delta_{\max }\right.$ for small deflections). The following paragraphs describe how the function $\delta_{\min }(N)$ has been deduced.

The function $\delta_{\min }(N)$ depends on the chosen dependence between the severity of the load, $\mathcal{G}_{\max }$ normalized to $\mathcal{G}_{c}$, and the crack length. We have chosen a linear decreasing dependence, from the normalized $\mathcal{G}_{\max , 0}$ at $a_{0}$ to $\mathcal{G}_{\max , f}$ at $a_{f}$, the end of the test (figure 3 ); however, other alternative monotonic dependence could be selected. Therefore, the gradient of the energy release rate, $\mathrm{d} \mathcal{G}_{\max } / \mathrm{d} a$, is constant and negative. Thus, the load severity vs. the crack length, $a$, reads: 


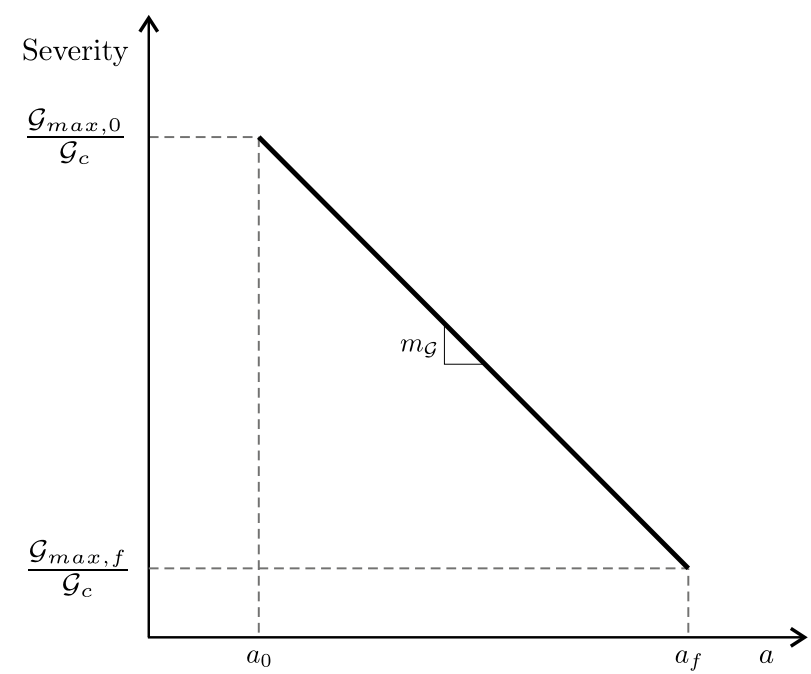

Figure 3: Chosen relation between the maximum cyclic energy release rate, $\mathcal{G}_{\max }$, normalized to the quasistatic fracture toughness, $\mathcal{G}_{c}$, and the crack length. $\mathcal{G}_{\text {max }, 0}$ and $a_{0}$ are the initital energy release rate and crack length, while $\mathcal{G}_{\max , f}$ and $a_{f}$ are the energy release rate and crack length at the end of the test.

$$
\frac{\mathcal{G}_{\max }}{\mathcal{G}_{c}}(a)=m_{\mathcal{G}} a+n
$$

where $m_{\mathcal{G}}$ and $n$ are the slope and the $y$-intercept, respectively:

$$
\begin{gathered}
m_{\mathcal{G}}=\frac{\mathcal{G}_{\max , f}-\mathcal{G}_{\max , 0}}{\mathcal{G}_{c}\left(a_{f}-a_{0}\right)}, \\
n=\frac{\mathcal{G}_{\max , 0}}{\mathcal{G}_{c}}-m_{\mathcal{G}} a_{0},
\end{gathered}
$$

Assuming a linear elastic behavior of the specimen, the energy release rate reads [24]:

$$
\mathcal{G}=\frac{P^{2}}{2 B} \frac{d C}{d a},
$$

94 thus, the minimum cyclic displacement $\left(\delta_{\text {min }}\right)$ is related to $\mathcal{G}_{\max }$ by

$$
\delta_{\text {min }}=-\sqrt{\frac{2 B \mathcal{G}_{\max } C^{2}}{\mathrm{~d} C / \mathrm{d} a}},
$$


where $B$ is the width of the specimen and $C$ is the specimen's compliance $(\delta / P$, where $P$ is the load).

The compliance of the specimen increases as the crack length grows. The dependence $C(a)$ is experimentally determined by a series of static tests at different crack lengths (compliance calibration)[19, 25]:

$$
C(a)=m_{c c} a^{3}+C_{0}
$$

where $m_{c c}$ and $C_{0}$ are fitting parameters.

Equations 1 to 6 can be used to write the dependence of $\left(\delta_{\min }\right)$ with the specimen compliance measured in real time:

$$
\delta_{\text {min }}(C)=-\sqrt{\frac{2 B \mathcal{G}_{c}\left[m_{\mathcal{G}}\left(\frac{C-C_{0}}{m_{c c}}\right)^{1 / 3}+n\right]}{3 m_{c c}\left(\frac{C-C_{0}}{m_{c c}}\right)^{2 / 3}}},
$$

Following this equation, the load severity would sweep the desired range (figure 3 ). The compliance can be easily measured in real time with current computerized testing systems, [11]. However, the control of the displacement based on the measurement of the compliance and equation 7 is problematic because of the inherent scatter of the experimental measurement, $C$, and the lack of data at the initiation of the test. It is thus preferable to base the control of the test on a certainly well-behaved variable, as the number of cycles, $N$, is.

To deduce the $\delta_{\min }(N)$ function, a relation between the crack length (or, equivalently, the compliance, equation 6) and the number of cycles is necessary. Here, we assume that the crack growth rate follows the Paris' law based expression [26]:

$$
\frac{\mathrm{d} a}{\mathrm{~d} N}=A\left(\frac{\mathcal{G}_{\max }}{\mathcal{G}_{c}}\right)^{p}
$$

Although the simplest expression of the Paris like power law has been used to relate crack growth to the energy release rate, it is worth noting that the methodology described here can also be used with other fatigue data representations based on the existing formulation for metals, such as the NASGRO 
where

$$
a(N)=\frac{(\alpha N+\beta)^{\gamma}-n}{m_{\mathcal{G}}},
$$

$$
\beta=\left(m_{\mathcal{G}} a_{0}+n\right)^{1-p},
$$

125 and

$$
\gamma=\frac{1}{1-p} .
$$

Finally, by substituting equations 1,9 , and 6 and its derivative, into equation 5 , the functions of ${ }_{127}$ the minimum cyclic displacement with the number of cycles reads: 


$$
\delta_{\min }(N)=-\sqrt{\frac{2 B \mathcal{G}_{c}(\alpha N+\beta)^{\gamma}\left(m_{c c}\left(\frac{(\alpha N+\beta)^{\gamma}-n}{m_{\mathcal{G}}}\right)^{3}+C_{0}\right)^{2}}{3 m_{c c}\left(\frac{(\alpha N+\beta)^{\gamma}-n}{m_{\mathcal{G}}}\right)^{2}}} .
$$

Hence, the minimum cyclic displacement is a function of the number of cycles $(N)$, the specimen width $(B)$, the initial conditions $\left(a_{0}, \mathcal{G}_{\max , 0} / \mathcal{G}_{c}\right)$, the user-defined gradient of energy release rate $\left(m_{\mathcal{G}}\right)$, the static compliance calibration parameters obtained prior to the fatigue test $\left(m_{c c}, C_{0}\right)$ and, finally, the Paris' law parameters $(A, p)$. In all events, $\delta_{\min }(N)$ is calculated previous to the fatigue test, so that the displacement can be automatically shed in a continuous manner by implementing equation 13 in the control software of the testing machine.

In this work it is assumed that the fatigue delamination growth, under pure mode II loading conditions, depends only on the peak energy release rate, $\mathcal{G}_{\max }$, and the load ratio (equivalent to the minimum to maximum cyclic displacement ratio, $R=\delta_{\min } / \delta_{\max }$, for small deflections). That is, it is assumed that the crack growth rate is neither history dependent nor dependent on the crack length, which is in agreement with other experimental evidence obtained from $\mathcal{G}_{\text {max }}$-constant tests with carbon/epoxy composites $[4,17,19]$.

\section{Experimental}

The validity of the test method to characterize mode II delamination growth was evaluated by comparing the crack growth rate curves obtained in variable (described in the previous section) and constant cyclic displacement tests. As the range of crack growth was very narrow in the latter case, a multiplicity of constant cyclic displacement tests were performed. The Paris' law parameters were determined by both methods. In addition, this exemplification allowed for a detailed comparison to be made of the effort saved by following the new experimental methodology proposed in this paper.

The laminates were 16 unidirectional carbon fiber/epoxy prepreg plies of $0.184 \mathrm{~mm}$ of nominal thickness stacked with the same fiber orientation $\left[0^{\circ}\right]$. Panels were cured in an autoclave, following the supplier's recommendations, at AERNNOVA Engineering facilities. Before cutting the specimens, 
the panels were ultrasonically C-scanned. 3-ENF test specimens cut from these laminates were $25 \mathrm{~mm}$ wide, $3 \mathrm{~mm}$ thick and $200 \mathrm{~mm}$ long. A $30 \mu \mathrm{m}$ thick and $60 \mathrm{~mm}$ length Teflon insert was introduced in the mid plane to create an artificial delamination. This teflon film was thicker than that usually recommended for static testing [29]; however, precracking was expected to avoid any possible negative effect resulting from the insert $[30,31]$. Precracks were performed under mode I quasi-static loading conditions until the increment in crack length was between $3 \mathrm{~mm}$ and $5 \mathrm{~mm}$. The teflon film was removed from the crack after the precracking procedure.

All the tests were carried out in a servohydraulic MTS Bionix ${ }^{\circledR}$ testing machine $(25 \mathrm{kN}$ of load capacity) under displacement control. The total force carried by the test specimen was measured with a $5 \mathrm{kN}$ MTS load cell. The three-point bending rig used to perform the tests met the specifications described in the ASTM standard for static testing [29]. The support rollers were $5 \mathrm{~mm}$ in radius, each with a span length between them of $100 \mathrm{~mm}$. Some tests under constant cyclic displacement conditions were performed with a span length between supports of $120 \mathrm{~mm}$ (longer than the standard), to enlarge the range for crack extension (see table 1). Tests were performed at the mechanical testing laboratory of the University of Girona, which is Nadcap [32] (Non-metallic materials testing laboratory) and ISO17025 [33] accredited.

The fatigue tests were performed under displacement control, by applying a sinusoidal waveform at a frequency of $5 \mathrm{~Hz}$ and setting the ratio of minimum to maximum displacement per cycle $(R)$ to $-10 /(-3)$. The desired severity at the beginning of the test was defined by the ratio of the initial maximum cyclic energy release rate $\left(\mathcal{G}_{\max , 0}\right)$ to the mean value of critical energy release rate $\left(\mathcal{G}_{c}\right)$ measured by quasi-static tests carried out prior to the fatigue test on identical test specimens. Assuming that the behavior of the specimen was linear elastic, the selection of the initial minimum displacement $\left(\delta_{\min , 0}\right)$ was related to $\mathcal{G}_{\max , 0}$ using equations 5 , and 6 and its derivative, and the initial crack length $\left(a_{0}\right)$ :

$$
\delta_{\min , 0}=-\frac{\left(m_{c c}\left(a_{0}\right)^{3}+C_{0}\right)}{a_{0}} \sqrt{\frac{2 B \mathcal{G}_{\max , 0}}{3 m_{c c}}}
$$


We established a maximum initial severity, $\mathcal{G}_{\max , 0} / \mathcal{G}_{c}$, of 0.55 to avoid the horizontal movement of the sample occurring for displacements below $-3.5 \mathrm{~mm}$. Other authors use mechanical restraints to avoid this [10] although this could affect the results.

The TestStar v3.5C control software for the MTS servohydraulic testing machine includes a "Calculated Channels" option that allows for internal variables, either external inputs or calculated through simple arithmetic operations, to be generated. It was used to compute the dynamic compliance, $C^{*}$, by processing the instantaneous signals of load and displacement in line with the methodology described in [11]. Next, the crack length was derived from the dynamic compliance using equation 6 :

$$
a=\sqrt[3]{\frac{C^{*}-C_{0}}{m_{c c}}}
$$

One set of data (the dynamic compliance, $C^{*}$, the minimum cyclic load, $P_{\min }$, and the number of cycles, $N$ ) was recorded every cycle and, as such, the crack length was calculated at the same monitoring frequency leading to a continuous $a(N)$ curve. Subsequently, the crack growth rate $(\mathrm{d} a / \mathrm{d} N)$ was obtained from the numerical derivative of the $a(N)$ curve. The recommended data reduction techniques for the ASTM standard [21] are the secant and the incremental polynomial methods. However, when these methodologies are applied to high frequency data acquisition curves, errors can occur because the dynamic compliance scatter might be too large compared to the increment in number of cycles between two successive data. For this reason, in this work the derivative was performed by linear regression of wider data sets, grouped so that the total increment of crack extension of each set was $0.1 \mathrm{~mm}[34]$.

Finally, $\mathrm{d} a / \mathrm{d} N$ was referred to the maximum energy release rate of the cycle normalized to the quasi-static critical value $\left(\mathcal{G}_{\max } / \mathcal{G}_{c}\right)$, or load severity. Taking equation 4 and the derivative of the compliance calibration $(6), \mathcal{G}_{\max }$ reads:

$$
\mathcal{G}_{\text {max }}=\frac{3 m_{c c} P_{\min }^{2} a^{2}}{2 B},
$$




\begin{tabular}{ccccc}
\hline \hline Specimen ID & $\begin{array}{c}\text { Initial load } \\
\text { severity } \\
\mathcal{G}_{\max , 0} / \mathcal{G}_{c}[-]\end{array}$ & $\begin{array}{c}\text { Initial crack length } \\
a_{0}[\mathrm{~mm}]\end{array}$ & $\begin{array}{c}\text { Mid-span length } \\
L[\mathrm{~mm}]\end{array}$ & $\begin{array}{c}\text { Initial minimum } \\
\text { displacement } \\
\delta_{\text {min }, 0}[\mathrm{~mm}]\end{array}$ \\
\hline 01_V & 0.50 & 30.0 & 50.00 & -3.108 \\
02_V & 0.50 & 35.0 & 50.00 & -3.075 \\
03_V & 0.50 & 38.0 & 50.00 & -3.071 \\
04_C & 0.55 & 38.0 & 50.00 & -3.356 \\
05_C & 0.40 & 38.0 & 50.00 & -2.770 \\
06_C & 0.30 & 38.0 & 50.00 & -2.543 \\
07_C & 0.25 & 45.0 & 60.00 & -3.096 \\
08_C & 0.20 & 38.0 & 50.00 & -2.104 \\
09_C & 0.18 & 45.0 & 60.00 & -2.743 \\
10_C & 0.14 & 45.0 & 60.00 & -2.347 \\
11_C & 0.12 & 45.0 & 60.00 & -2.223 \\
\hline \hline
\end{tabular}

Table 1: Initial conditions for the propagation tests. In the specimen identification, "V" stands for variable displacement tests and "C" stands for constant displacement tests.

where the crack length, $a$, is taken at the midpoint of the cycle.

Constant displacement tests were performed using eight different initial severities in order to cover a wider portion of the $\mathrm{d} a / \mathrm{d} N$ curve and the results were fitted together using the modified Paris' law from equation 8 .

As mentioned in section 1 , the energy release rate does not evolve monotonically with the crack extension, but rather increases and then decreases under constant cyclic displacement, and with the maximum point (point 2 in figure 2a) always being located at $a=0.7 L$. Therefore, constant cyclic displacement tests were performed with initial crack length higher than $0.7 L$, in order to avoid sweeping the same part of the crack growth rate curve twice (see figure $2 \mathrm{~b}$ ). In contrast, in variable cyclic displacement tests the initial crack length is not a limiting parameter because the dependence between the load severity and the crack length is monotonic. In this specific case, the authors chose different crack lengths in order to analyze the influence of the initial conditions on the resultant crack growth rate curve.

Table 1 indicates the initial conditions for each propagation test. The specimens labelled "V" were tested by applying the variable cyclic displacement methodology presented in this work and the specimens labelled "C" were tested under constant cyclic displacement conditions.

For the variable cyclic displacement tests, the "Calculated Channels" from the MTS TestStar v3.5C 


\begin{tabular}{|c|c|c|c|c|c|c|c|c|}
\hline \multirow[t]{2}{*}{$\begin{array}{l}\text { Specimen } \\
\text { ID }\end{array}$} & \multirow{2}{*}{$\begin{array}{c}\begin{array}{c}\text { Specimen } \\
\text { width }\end{array} \\
\\
B \\
{[\mathrm{~mm}]}\end{array}$} & \multirow{2}{*}{$\begin{array}{c}\begin{array}{c}\text { Initial } \\
\text { crack } \\
\text { length } \\
\\
\\
a_{0} \\
{[\mathrm{~mm}]}\end{array}\end{array}$} & \multicolumn{2}{|c|}{$\begin{array}{c}\text { Estimated fatigue } \\
\text { life constants } \\
\text { (eq. 8), } \\
\mathrm{d} a / \mathrm{d} N \text { evaluated } \\
\text { in } \mathrm{mm} / \text { cycle }\end{array}$} & \multicolumn{2}{|c|}{$\begin{array}{c}\text { Compliance calibration } \\
\text { parameters } \\
\text { (eq. 6) }\end{array}$} & \multicolumn{2}{|c|}{$\begin{array}{l}\text { Severity versus } \\
\text { crack length } \\
\text { relation constants } \\
\text { (eq. 1) }\end{array}$} \\
\hline & & & $A$ & $p$ & $\begin{array}{c}m_{c c} \\
{\left[\mathrm{~N}^{-1} \mathrm{~mm}^{-2}\right]}\end{array}$ & $\begin{array}{c}C_{0} \\
{\left[\mathrm{~mm} \mathrm{~N}^{-1}\right]}\end{array}$ & $\begin{array}{c}m_{\mathcal{G}} \\
{\left[\mathrm{mm}^{-1}\right]}\end{array}$ & $\begin{array}{c}n \\
(-)\end{array}$ \\
\hline $01 \_\mathrm{V}$ & 25.00 & 30.0 & $6.19210^{-2}$ & 3.674 & $3.19310^{-8}$ & $2.60810^{-3}$ & -0.030 & 1.400 \\
\hline $02 \_\mathrm{V}$ & 25.00 & 35.0 & $5.59510^{-2}$ & 3.695 & $3.13110^{-8}$ & $2.62610^{-3}$ & -0.090 & 3.650 \\
\hline $03 \_\mathrm{V}$ & 25.00 & 38.0 & $5.59510^{-2}$ & 3.695 & $3.18810^{-8}$ & $2.59410^{-3}$ & -0.225 & 9.050 \\
\hline
\end{tabular}

Table 2: Parameters used in the minimum variable displacement calculation, $\delta_{m i n}$, using equation 13.

software allowed an internal variable to be calculated (in accordance with equation 13) in real time. This variable was established as the continuous displacement setpoint, $\delta_{\min }$.

Table 2 lists the parameters used for calculating of $\delta_{\min }(N)$ (equation 13). The Paris' law parameters, $A$ and $p$, obtained in the d $a / \mathrm{d} N$ data fitting resulting from tests $07 \_\mathrm{C}, 09 \_\mathrm{C}, 10 \_\mathrm{C}$ and 11_C, were used to estimate the curve $\delta_{\min }(N)$ for test $01 \_V$. The results from tests $06 \_\mathrm{C}$ and $08 \_\mathrm{C}$ were added to the previous data to obtain $A$ and $p$ for tests $02 \_\mathrm{V}$ and $03 \_\mathrm{V}$.

Constant cyclic displacement tests were performed on specimens with a mode I precrack, whereas the variable cyclic displacement tests were performed on specimens already tested under constant cyclic displacement (thus having a mode II fatigue pre-crack). The reason for this was to avoid the transient behavior observed at the onset of delamination for specimens with a mode I precrack, as described further in sections 4 and 5 .

\section{Results}

A typical crack growth rate curve $(d a / d N$ vs severity) in a specimen precracked under mode I and loaded under constant displacement amplitude exhibits three distinct stages (figure 4). As the severity decreases from the onset of the test, the curve sweeps from right to left. The first region is characterized by a growing crack growth rate as the severity decreases. Then, the largest region (i.e. the second region), consists of a smooth direct dependence between $d a / d N$ and severity. Finally, in 
the third region the crack tends to arrest with a higher slope than that seen in the second region. For these reasons discussed in the next section, the curve was truncated, neglecting the first and third regions. Only the mid-region was considered in the modified Paris' law calculation.

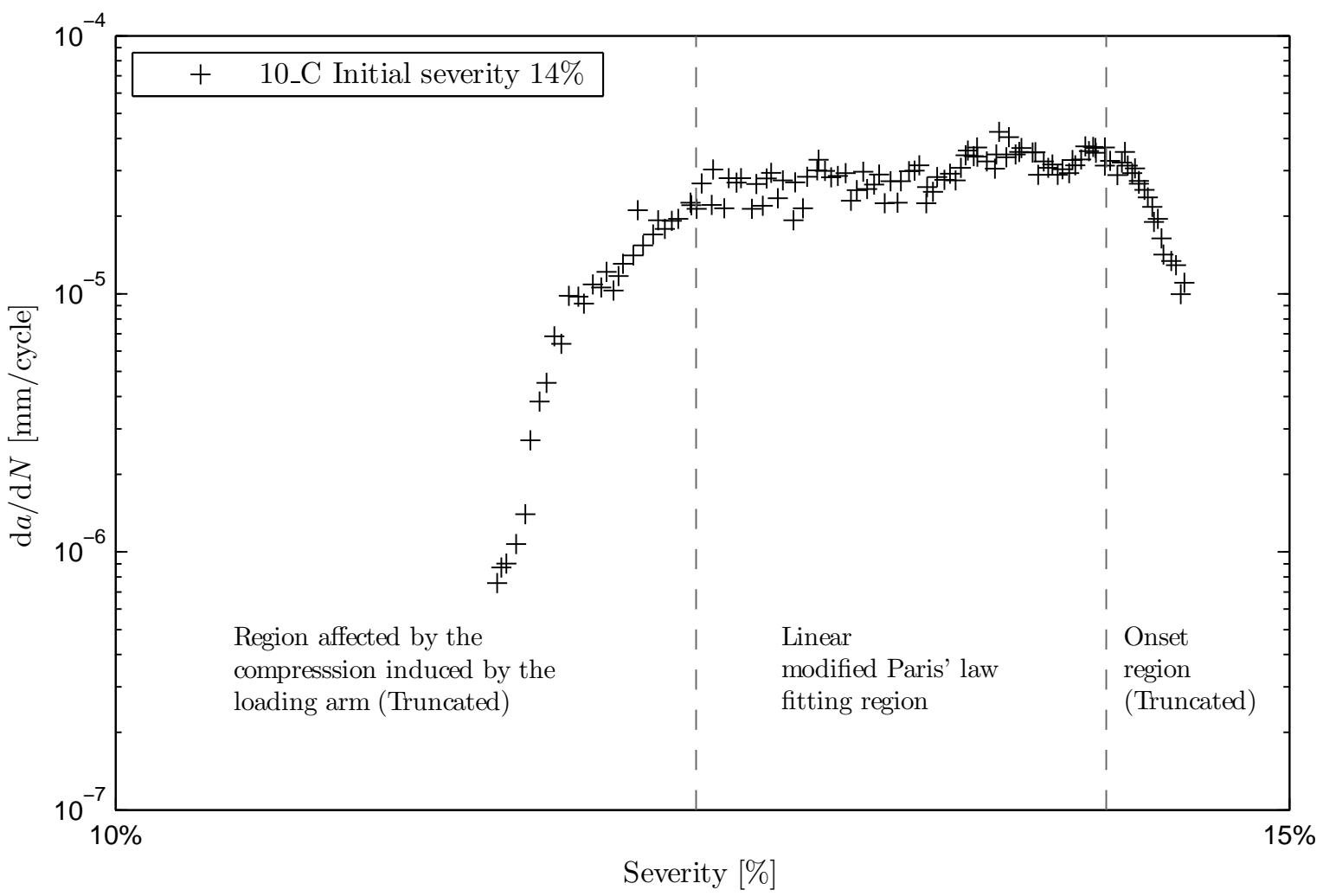

Figure 4: Reduced fatigue crack growth rate data with the truncated regions in the modified Paris' law fitting from the constant cyclic displacement tests.

As variable displacement tests were performed on specimens already tested under mode II, their crack growth rate curves did not show the first region of figure 4 . Figure 5 compares the preselected and the experimental dependence between the severity and the crack length increment, confirming the new experimental methodology as being suitable to enlarge the range of severities explored in a single test.

The crack growth rate curve in figure 6 condensates the results obtained from the eleven tests performed in this study. All the tests were performed for the same load ratio, $R=-10 /(-3)$, either under constant cyclic displacement or variable cyclic displacement control. The plot also includes the 


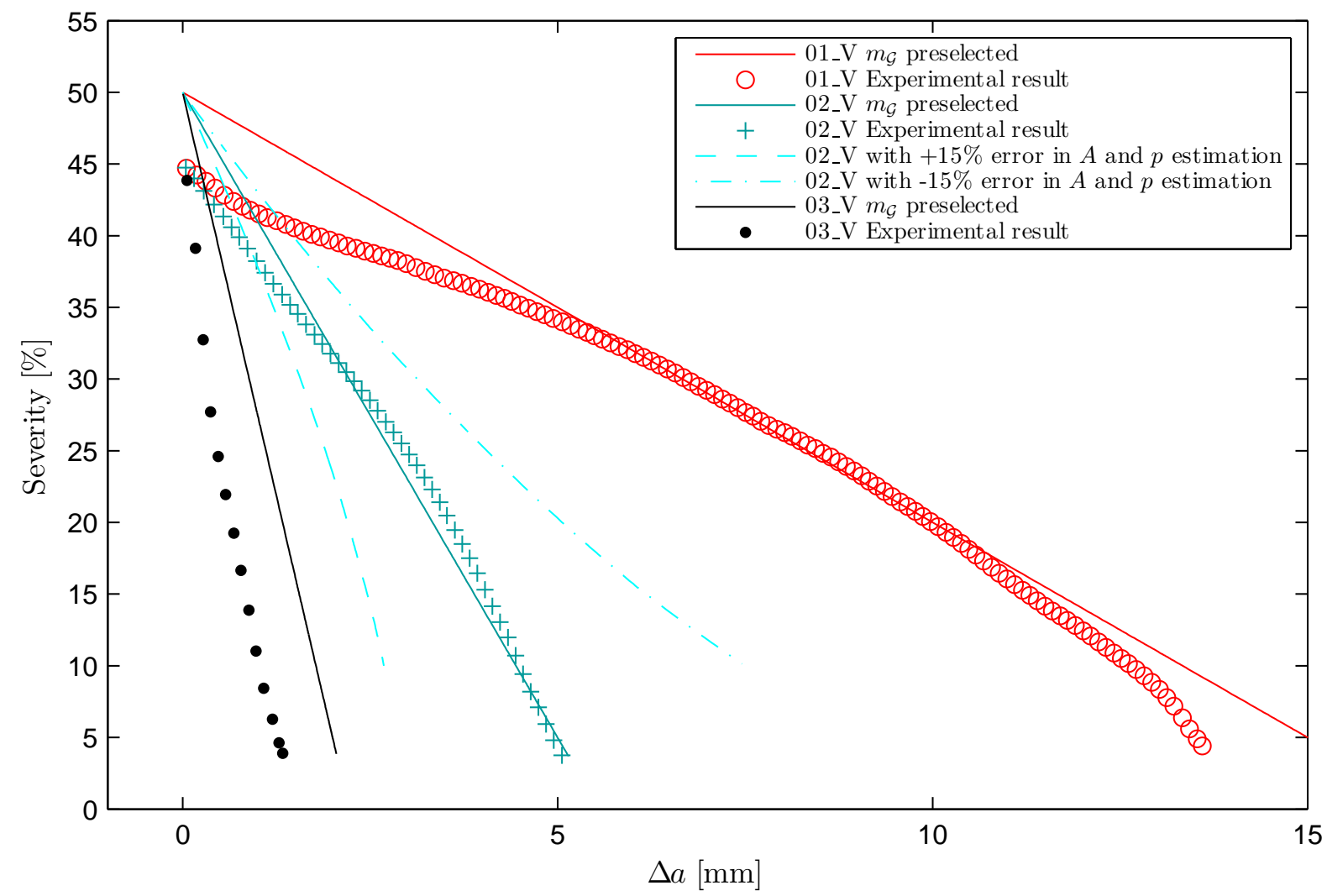

Figure 5: Comparison between the preselected slope, $m_{\mathcal{G}}$, from the energy release rate versus the crack length curve and the experimental relationship $\frac{\mathcal{G}_{\max }}{\mathcal{G}_{c}}-\Delta a$ obtained from the variable displacement tests. Dashed lines illustrate the $\frac{\mathcal{G}_{\max }}{\mathcal{G}_{c}}-\Delta a$ relation obtained if the Paris' law parameters, $A$ and $p$, used in the calculation of $\delta_{\min }(N)$ in test $02{ }_{-} \mathrm{V}$, were predicted with an error of $\pm 15 \%$.

fitting of all the data from the constant cyclic displacement tests in accordance with the modified

Paris' law (equation 8).

The parameters of the modified Paris' law (equation 8), the exponent, $p$, and the coefficient, $A$, are obtained from the linear fitting of the data plotted on log-log scales (table 3).

The duration of each variable cyclic displacement test depends on the slope of the relation between the energy release rate and crack length, $m_{\mathcal{G}}$ (figure 5 ). Higher slopes tend to minimize the testing time. The total time employed in all tests (constant displacement and variable displacement) is specified in table 4 . 


\begin{tabular}{cccc}
\hline \hline Specimen ID & $\begin{array}{c}\text { Severity versus } \\
\text { crack length } \\
\text { slope (eq. 1) }\end{array}$ & $\begin{array}{c}\text { Obtained fatigue life constants } \\
\text { (eq. 8), } \mathrm{d} a / \mathrm{d} N \text { evaluated in } \\
\mathrm{mm} / \text { cycle }\end{array}$ \\
\cline { 2 - 4 } & $\begin{array}{c}m_{\mathcal{G}} \\
{\left[\mathrm{mm}^{-1}\right]}\end{array}$ & $A$ & $p$ \\
\hline $01 \_\mathrm{V}$ & -0.030 & $1.97510-1$ & 4.326 \\
$02 \_\mathrm{V}$ & -0.090 & $1.67710-1$ & 4.168 \\
$03 \_\mathrm{V}$ & -0.225 & $7.34210-2$ & 3.786 \\
\hline Modified Paris' law (Eq. 8) fitting of the & & \\
constant displacement tests' results & $7.63610-2$ & 3.882 \\
\hline \hline
\end{tabular}

Table 3: Fatigue life constants obtained from both variable displacement and constant displacement tests.

\begin{tabular}{clcc}
\hline \hline & Specimen ID & $\begin{array}{c}\text { Total time } \\
\text { (hours) }\end{array}$ & $\begin{array}{c}\text { Time 10-45\% severity } \\
\text { (hours) }\end{array}$ \\
\hline \multirow{3}{*}{ Variable displacement tests } & 01_V Severity from 50\% to 10\% & 42.0 & 4.3 \\
& 02_V Severity from 50\% to 10\% & 18.0 & 1.9 \\
& 03_V Severity from 50\% to 10\% & 7.0 & 0.4 \\
\hline & 04_C Initial severity 55\% & 0.2 & \\
& 05_C Initial severity 40\% & 1.3 & \\
& 06_C Initial severity 30\% & 2.6 & \\
Constant displacement tests & 07_C Initial severity 25\% & 4.0 & \\
& 08_C Initial severity 20\% & 8.8 & \\
& 09_C Initial severity 18\% & 15.5 & \\
& 10_C Initial severity 14\% & 48.1 & \\
\hline Total time employed in & 11_C Initial severity 12\% & 89.1 & \\
constant displacement testing & & & \\
\hline \hline
\end{tabular}

Table 4: Time employed in obtaining the crack growth rate curve for both variable and constant minimum displacement methodologies. 


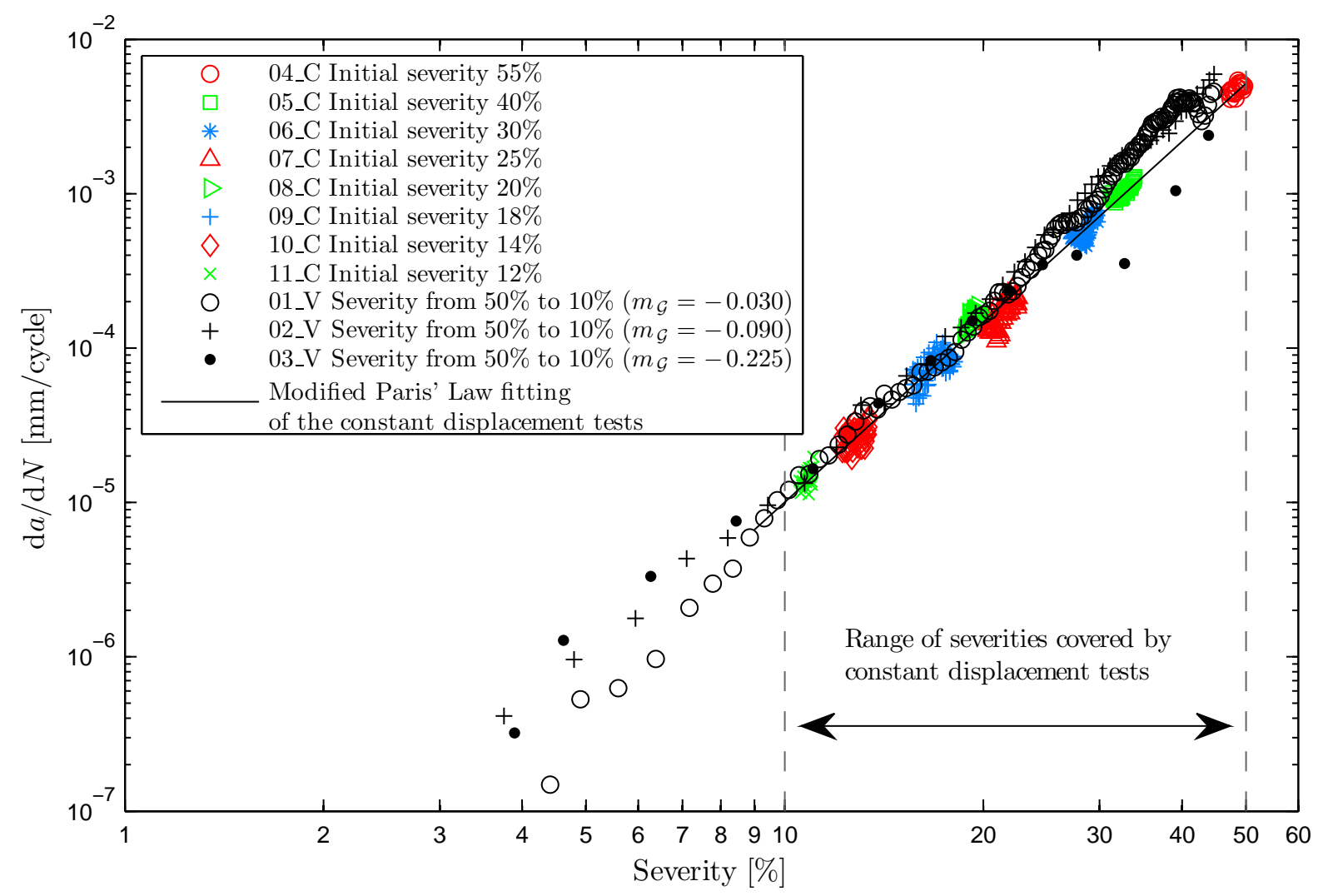

Figure 6: Relation between crack propagation rate and peak energy release rate for $R=0.3$. The results from both variable displacement ("V" labelled) and constant displacement ("C" labelled) tests are presented for comparison proposes.

\section{Discussion}

The proposed experimental methodology for mode II testing in the 3-ENF configuration enables a chosen range of load severities to be swept while the crack grows in a predefined crack length increment. This is accomplished by varying the applied cyclic displacement as the number of cycles evolves. The $\delta_{\text {min }}(N)$ function depends on estimated Paris' law parameters $(A$ and $p)$, derived from the constant cyclic displacement tests. In spite of being just a rough estimation, the range of severities swept is close to the desired one and, in any case, much larger than what could be obtained from a constant displacement test. The consequence of using erroneous parameters is illustrated in figure 5 for the specimen $02 \_V$. An error in $A$ and $p$ of $\pm 15 \%$ leads to changes in the crack length increment needed to sweep the desired severity range. In any event, the severity range achieved would be much larger 
than that attained using a constant displacement test.

The variable cyclic displacement method leads to crack growth rate data practically indistinguishable from that resulting from the complete set of eight constant displacement tests. In particular, the Paris' exponent, $p$, obtained from variable cyclic displacement tests under steady growth conditions (severities from 10 to $30 \%$ ), deviated from the log-linear fitting of the constant cyclic displacement tests results by $+11.4 \%,+7.4 \%$ and $-2.5 \%$ (specimens $01 \_\mathrm{V}, 02 \_\mathrm{V}$ and $03 \_\mathrm{V}$, respectively). No experimental data for severities higher than $45 \%$ could be obtained because of specimen horizontal movement. The agreement between the results from both methods, however, is expected to persist if this issue could be solved practically.

One of the main advantages of the new experimental methodology is the reduction of the time duration of the fatigue mode II test. The selection of the shedding rate, $m_{\mathcal{G}}$, determines the duration of the test. Table 4 exemplifies the time saved with this method. One single test performed with variable displacement to build the crack growth rate curve for a severity range between $10 \%$ and $45 \%$ is performed in $1.9 \mathrm{~h}$ (specimen $02 \_\mathrm{V}$ ), while the 8 constant displacement tests needed to sweep the same severity range require $169.6 \mathrm{~h}$ (80 times more if the times involved in setting up each of the tests is not considered).

The question arises of how fast the test can be carried out while still leading to the crack growth curve obtained in a constant displacement test. This question could be dealt with taking into account the formation of a failure process zone, FPZ, in front of the crack tip. While the FPZ for mode I delamination in CFRP is assumed to be so small as to be negligible in the data reduction of static interlaminar fracture toughness tests, the same does not apply for the FPZ in mode II tests [35]. Under mode II loading and for a given load severity, the crack growth rate would not reach its steady level until the FPZ is fully formed. In a variable displacement test, if the severity varies before the FPZ is fully formed, the crack growth rate would deviate from the steady crack growth rate for the actual severity being applied. This is more likely to happen as the shedding rate increases and that deviation would also be more important the stronger the variation of the FPZ with the load severity is. 
Figure 6 illustrates the crack growth rate curves obtained from the variable cyclic displacement tests for the three shedding rates, $m_{\mathcal{G}}$, explored in this study. The tests performed at low $m_{\mathcal{G}}$ lie close to the Paris' curve of constant cyclic displacement tests from the very first stages of the crack growth curve (higher severities), whereas the test performed at high $m_{\mathcal{G}}\left(03_{-} \mathrm{V}\right)$ tends to deviate from these curves in the region of high $(30-45 \%)$ and low $(<10 \%)$ severities, while in the region in between the agreement is complete. The deviation of specimen $03_{-} \mathrm{V}$ at the beginning of the test (high severities) corresponds to the transient stage from the FPZ of the precrack to the steady FPZ. On the contrary, at lower severities, below $10 \%$, the deviation is attributed to a shedding rate too fast to permit the stabilization of the FPZ, leading to a faster crack growth than observed in constant cyclic displacement tests. The confirmation of these hypothesis and the study of the FPZ zone in fatigue tests as a function of the load severity deserves further investigation.

These facts highlight the impact the pre-cracking stage has on the initial measurements of the crack growth rate curves. In the first stage of the crack growth curve for constant displacement tests in figure 4 there is a clear evidence of the transient region between the FPZ of the precrack and the steady FPZ. Indeed, due to the fact that the constant displacement tests start from a mode I precrack (short FPZ), the crack grows more slowly than the steady rate until the mode II FPZ is fully formed.

In view of the foregoing, the variable cyclic displacement tests were performed on specimens tested under mode II cyclic loading, where the crack grew until the tip reached the zone affected by loading arm compression ("Region affected by the compression induced by the loading arm" in figure 4). In this region, the crack propagation tends to arrest, misrepresenting an artificial threshold. The FPZ formed in this situation (low severity), however, is different from the one expected at the beginning of the variable displacement tests (high severity). For that reason, a transient region in the variable displacement tests is expected. While this is not noticeable in specimens with low $m_{\mathcal{G}}$, it does span over several points for specimen $03 \_V$.

The fact that the three crack growth curves obtained under variable cyclic displacement amplitude with different $m_{\mathcal{G}}$ coincide in the severity range between $30 \%$ and $10 \%$ indicates that the shedding 
rate is slow enough to lead to a fully developed FPZ, even with the highest $m_{\mathcal{G}}$ selected (figure 6). The prospect is that for a large enough $m_{\mathcal{G}}$, the crack growth curve would deviate from that of the constant displacement test. Following an equivalent rationale, the crack length increment can not be decreased arbitrarily if representative results are to be obtained. Figure 5 shows that the same Paris' law curve is obtained for crack increments of $2 \mathrm{~mm}$ (specimen 03_V) or $14 \mathrm{~mm}$ (specimen 01_V). These constraints should be specifically considered when testing materials with expected large FPZ, as the case of adhesive joints is. The larger the expected FPZ, the slower the shedding rate should be and, likewise, the larger the crack increment to be traveled.

The comparison of the results from constant cyclic displacement tests reveals that the propagation rate does not depend on the span length (e.g. specimens 07_C and 08_C, with span lengths of 60 and $50 \mathrm{~mm}$ respectively, have similar $\mathrm{d} a / \mathrm{d} N$ at $20 \%$ severity). Thus, the same results are obtained with different crack lengths. This is an indication that, for the material studied, the crack growth rate is history independent under mode II test conditions when a steady crack growth is achieved (initial transient curves must be truncated, figure 4). This assertion is corroborated by the overlap among the crack growth rate curves obtained with the three variable displacement tests performed with different preselected gradients of energy release rate, $m_{\mathcal{G}}$ (see table 3 ), again, once the FPZ is fully developed. Under this condition, the maximum difference in $\mathrm{d} a / \mathrm{d} N$, for a given severity, between the data obtained from variable displacement tests and the fitting of all the data from constant displacement tests, amounts 0.3 decades. This is comparable to the scatter of the raw data from a single constant displacement test.

\section{Conclusions}

An automated procedure to obtain the log-linear region of a crack growth rate curve has been developed for an 3-ENF test for mode II fatigue delamination growth. The displacement applied, $\delta(N)$, is calculated prior to initiating the fatigue test in order to achieve a constant negative energy release rate gradient throughout crack propagation. The continuous displacement shedding is conducted by 
implementing the calculated $\delta(N)$ curve in a computer-controlled testing system. This, in combination with the automated and continuous estimation of the crack length by means of the real time monitoring of the specimen's compliance, avoids the need of human intervention during the test.

The usefulness of this methodology has been exemplified with an experimental testing campaign in which the crack growth rate curve obtained is compared with the modified Paris' law fitting data from a batch of constant cyclic displacement tests.

The range of severities covered by a single test using the developed methodology spans from 0.45 to 0.1. Due to the specimen movement, the initial severity could not be higher than 0.50 .

The time saved employing the methodology developed has been demonstrated (the example performed shows a reduction of $1 / 80$ ) and how the duration of the test, which is determined by the shedding rate and the range of severities explored, is limited by the requirement of forming the complete failure process zone corresponding to the actual load severity is discussed.

\section{Acknowledgements}

This work has been partially funded by the Spanish Government (Ministerio de Economia y Competitividad) under contract TRA2015-71491-R and the European Union by the financial support of ERANet AirTN 01/2013 under the project entitled "Methodology to design composite structures resistant to intra- and interlaminar damage (static \& fatigue)- MERINDA".

\section{References}

[1] SAE International, Polymer Matrix Composites: Materials Usage, Design and Analysis, Composite Materials Handbook Series 3 (2013) 686-794.

[2] ASTM D 6115-97, Standard test method for mode I fatigue delamination growth onset of unidirectional fibre-reinforced polymer matrix composites (2011).

[3] C. Dahlen, G. S. Springer, Delamination Growth in Composites under Cyclic Loads, Journal of Composite Materials 28 (8) (1994) 732-781. 
[4] A. Argüelles, J. Viña, A. F. Canteli, J. Bonhomme, Fatigue Delamination, Initiation, and Growth, Under Mode I and II of Fracture in a Carbon-Fiber Epoxy Composite, Polymer Composites 31 (4) (2010) 700-706.

[5] G. Allegri, M. Jones, M. Wisnom, S. Hallett, A new semi-empirical model for stress ratio effect on mode II fatigue delamination growth, Composites Part A: Applied Science and Manufacturing $42(7)(2011) 733-740$.

[6] G. Allegri, M. R. Wisnom, A non-linear damage evolution model for mode II fatigue delamination onset and growth, International Journal of Fatigue 43 (2012) 226-234.

[7] G. Allegri, M. R. Wisnom, S. R. Hallett, A new semi-empirical law for variable stress-ratio and mixed-mode fatigue delamination growth, Composites Part A: Applied Science and Manufacturing 48 (1) (2013) 192-200.

[8] O. Al-Khudairi, H. Hadavinia, A. Waggott, E. Lewis, C. Little, Characterising mode I/mode II fatigue delamination growth in unidirectional fibre reinforced polymer laminates, Materials \& Design 66 (2015) 93-102.

[9] L. Asp, A. Sjogren, E. Greenhalgh, Delamination Growth and Thresholds in a Carbon/Epoxy Composite Under Fatigue Loading, Journal of Composites Technology and Research 23 (2) (2001) 55.

[10] A. Brunner, S. Stelzer, G. Pinter, G. P. Terrasi, Mode II fatigue delamination resistance of advanced fiber-reinforced polymer-matrix laminates: Towards the development of a standardized test procedure, International Journal of Fatigue 50 (2013) 57-62.

[11] J. Renart, J. Vicens, S. Budhe, J. Costa, J. A. Mayugo, An automated methodology for mode II delamination tests under fatigue loading based on the real time monitoring of the specimen's compliance, International Journal of Fatigue 82 (2016) 634-642. 
[12] ISO 15114:2014, Fibre-reinforced plastic composites Determination of the mode II fracture resistance for unidirectionally reinforced materials using the calibrated end-loaded split (C-ELS) test and an effective crack length approachs (2014).

[13] Y. Shindo, T. Takeda, F. Narita, N. Saito, S. Watanabe, K. Sanada, Delamination growth mechanisms in woven glass fiber reinforced polymer composites under Mode II fatigue loading at cryogenic temperatures, Composites Science and Technology 69 (200914) 1904-1911.

[14] B. D. Davidson, X. U. Sun, A. J. Vinciquerra, Influences of Friction, Geometric Nonlinearities, and Fixture Compliance on Experimentally Observed Toughnesses from Three and Four-point Bend End-notched Flexure Tests, Journal of Composite Materials 41 (10) (2007) 1177-1196.

[15] A. Brunner, N. Murphy, G. Pinter, Development of a standardized procedure for the characterization of interlaminar delamination propagation in advanced composites under fatigue mode I loading conditions, Engineering Fracture Mechanics 76 (2009) 2678-2689.

[16] J. W. Gillespie, L. A. Carlsson, R. B. Pipes, Finite Element Analysis of the End Notched Flexure Specimen for Measuring Mode II Fracture Toughness, Composites Science and Technology 27 (1986) 177-197.

[17] K. Tanaka, H. Tanaka, Stress-ratio effect on mode II propagation of interlaminar fatigue cracks in Graphite/Epoxy composites, Composite Materials: Fatigue and Fracture (Sixth volume), ASTM STP 1285 (1997) 126-142.

[18] G. Matsubara, H. Ono, K. Tanaka, Mode II fatigue crack growth from delamination in unidirectional tape and satin-woven fabric laminates of high strength GFRP, International Journal of Fatigue 28 (10) (2006) 1177-1186.

[19] M. Hojo, T. Ando, M. Tanaka, T. Adachi, S. Ochiai, Y. Endo, Modes I and II interlaminar fracture toughness and fatigue delamination of $\mathrm{CF} /$ epoxy laminates with self-same epoxy interleaf, International Journal of Fatigue 28 (10) (2006) 1154-1165. 
[20] M. Hojo, K. Tanaka, C. G. Gustafson, R. Hayashi, Effect of stress ratio on near-threshold propagation of delimination fatigue cracks in unidirectional CFRP, Composites Science and Technology 29 (1987) 273-292.

[21] ASTM E647-13, Standard Test Method for Measurement of Fatigue Crack Growth Rates, American Society for Testing and Materials (ASTM) (2013) 1-49.

[22] A. Saxena, D. Schmidt, J. Donald, S. Hudak, Computer-controlled decreasing stress intensity technique for low rate fatigue crack growth testing, Journal of Testing and Evaluation 6 (3) (1978) $167-174$

[23] D. Schmidt, J. Donald, Computer-controlled stress intensity gradient technique for high rate fatigue crack growth testing, Journal of Testing and Evaluation 8 (1) (1980) 19-24.

[24] T. L. Anderson, Fracture Mechanics: Fundamentals and Applications, 3rd Edition, CRC Press (2005).

[25] M. Hojo, S. Matsuda, B. Fiedler, T. Kawada, K. Moriya, S. Ochiai, H. Aoyama, Mode I and II delamination fatigue crack growth behavior of alumina fiber/epoxy laminates in liquid nitrogen, International Journal of Fatigue 24 (2002) 109-118.

[26] P. C. Paris, A rational analytic theory of fatigue, The Trend in Engineerging 13 (1961) 9-14.

[27] F. G. Forman, V. Shivakumar, J. W. Cardinal, L. Williams, P. C. McKeighan, Fatigue Crack Growth Database for Damage Tolerance Analysis, DOT/FAA/AR-05/15. US Department of Transportation, Federal Aviation Administration (2005).

[28] R. Jones, S. Stelzer, A. J. Brunner, Mode I, II and Mixed Mode I/II delamination growth in composites, Composite Structures 110 (2014) 317-324.

[29] ASTM D7905/7905M-14, Standard Test Method for Determination of the Mode II Interlaminar Fracture Toughness of Unidirectional Fiber-Reinforced Polymer, American Society for Testing and Materials (ASTM) (2014) 1-18. 
[30] K. Tanaka, K. Kageyama, M. Hojo, Prestandardization study on mode II interlaminar fracture toughness test for CFRP in japan, Composites 26 (4) (1995) 257-267.

[31] JIS K 7086-1993, Testing Methods for Interlaminar Fracture Toughness of Carbon Fibre Reinforced Plastics, Japanese Industrial Standards Group.

[32] AC7122/1 Rev B, Nadcap Audit Criteria for Non Metallic Materials Testing Mechanical Testing.

[33] ISO/IEC 17025, General requirements for the competence of testing and calibration laboratories (2014).

[34] S. Stelzer, A. J. Brunner, A. Argüelles, N. Murphy, G. M. Cano, G. Pinter, Mode I delamination fatigue crack growth in unidirectional fiber reinforced composites : Results from ESIS TC4 roundrobins, Engineering Fracture Mechanics 116 (2014) 92-107.

[35] C. Sarrado, A. Turon, J. Renart, I. Urresti, Assessment of energy dissipation during mixed-mode delamination growth using cohesive zone models, Composites Part A 43 (11) (2012) 2128-2136. 